\title{
High-Resolution Infrared and Raman Spectra of the Polycrystalline Sinomenine Hydrochloride
}

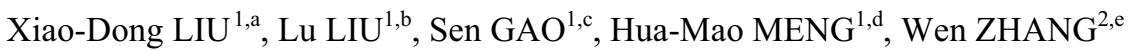 \\ ${ }^{1}$ School of Science, Tianjin Polytechnic University, Tianjin 300387, China \\ ${ }^{2}$ School of Material Science and Engineering, Tianjin Polytechnic University, Tianjin 300387, China \\ aliuxiaodong@tjpu.edu.cn, ${ }^{b} 15202203136 @ 163 . c o m,{ }^{c} 393495891 @ q q . c o m,{ }^{d} h u a m a o 254 @$ sohu.com, \\ 'zhangwen2050@hotmail.com
}

\begin{abstract}
High-resolution infrared and Raman spectra of the polycrystalline sinomenine (SM) hydrochloride have been measured to work out its whole really existing vibrational spectral bands. Except for the hydroxyl stretching modes and IR active bands less than $400 \mathrm{~cm}^{-1}$, most normal modes (about 34) are both IR and Raman active. In addition, 8 Raman bands less than $400 \mathrm{~cm}^{-1}$ are tentatively assigned, for the first time to our knowledge, to stretching/bending modes of the aromatic-ring-methoxyls and $(\mathrm{SMH})^{+}-\mathrm{Cl}^{-}$ions, respectively.
\end{abstract}

\section{Introduction}

The sinomenine hydrochloride $\left(\mathrm{C}_{19} \mathrm{H}_{24} \mathrm{NO}_{4} \mathrm{Cl}\right.$, abbreviated $\left.\mathrm{SMHCl}\right)$ is the hydrochloric acid compound of the sinomenine $\left(\mathrm{C}_{19} \mathrm{H}_{23} \mathrm{NO}_{4}\right.$, abbreviated $\left.\mathrm{SM}\right)$, which is a chemical compound possessing important medical values and initially found to exist in traditional Chinese medicinal materials [1-3], and is prone to crystallizing to form the polycrystalline unit. Therefore its vibrational spectral analysis becomes extremely useful, for example, the spectral analysis can be used to anlysize the chemical purity and the hydrochlorination level for a given $\mathrm{SMHCl}$ sample or the structural modification of the SM derivatives [4-6].

Whereas the $\mathrm{SMHCl}$ molecule consists of many vibrational groups, and has many normal lattice vibrational modes [7]. Here, we try to work out the whole really existing vibrational spectral bands of polycrystalline $\mathrm{SMHCl}$ with the high-resolution infrared (IR) spectrometer and laser micro-Raman spectroscopy.

\section{Experimental methods}

The polycrystalline $\mathrm{SMHCl}$ with the purity of more than $99 \%$ was purchased from Xi' an Feida biotechnology co., ltd, Xi'an, Shanxi Province, China.

* Corresponding author:liuxiaodong@tjpu.edu.cn 
The IR spectrum of the polycrystalline $\mathrm{SMHCl}$ was measured with the Thermo Scientific Nicolet iS50 spectrometer whose resolution is high up to $<0.09 \mathrm{~cm}^{-1}$ by using of the general $\mathrm{KBr}$ disc technology in the range of $3600-400 \mathrm{~cm}^{-1}$. Raman spectrum was obtained using Thermo Scientific DXR Raman microscope excited by an Nd: YAG laser (532.0 nm @ 5 $\mathrm{mW})$ at a resolution of $1.5 \mathrm{~cm}^{-1}(900 \mathrm{~g} / \mathrm{mm})$ between $3600-50 \mathrm{~cm}^{-1}$. The Raman spectrum was automatically baseline corrected (fluorescence subtracted partly), moderately smoothed and normalized according to the largest counting peak value.

\section{Results and discussion}

Figures 1 3 show the IR and Raman spectra of the polycrystalline SMHCl in the ranges of $3600-2800 \mathrm{~cm}^{-1}, 1750-900 \mathrm{~cm}^{-1}$ and $900-50 \mathrm{~cm}^{-1}$, respectively, where blue wavenumbers represent the both-IR-and-Raman-active modes whose differences of corresponding wavenumbers are between 10 and $-10 \mathrm{~cm}^{-1}$.
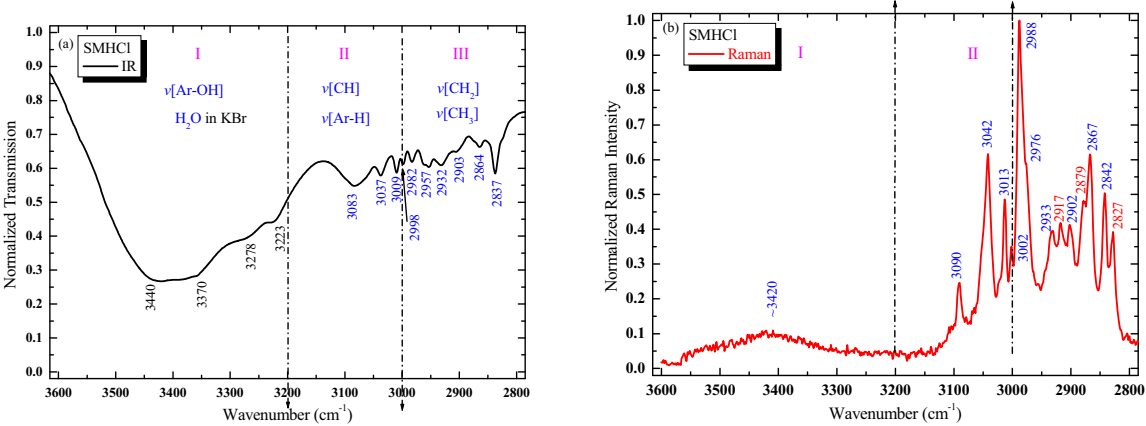

Fig. 1. IR (a) and Raman (b) spectra of the polycrystalline sinomenine hydrochloride ( $\left.\mathrm{SMHCl}, \mathrm{C}_{19} \mathrm{H}_{24} \mathrm{NO}_{4} \mathrm{Cl}\right)$ in the range of $3600-2800 \mathrm{~cm}^{-1}$ (the $[R \mathrm{H}]$ functional group region).

From Fig. 1, one can see that the SMHCl sample contains same crystal water which, together with the water in $\mathrm{KBr}$, have produced $\mathrm{O}-\mathrm{H} \cdots \mathrm{O}$ hydrogen bond and caused a decreased $v[\mathrm{Ar}-\mathrm{OH}]$ in the IR spectrum, but the corresponding Raman spectrum is greatly widened because of the laser local heating effect [8]. In the $[R \mathrm{H}]$, other than $[\mathrm{Ar}-\mathrm{OH}]$, functional groups region, all the ten modes found in the IR spectrum have their corresponding Raman ones, which are proved to be really existing and both IR and Raman active because of their asymmetry.
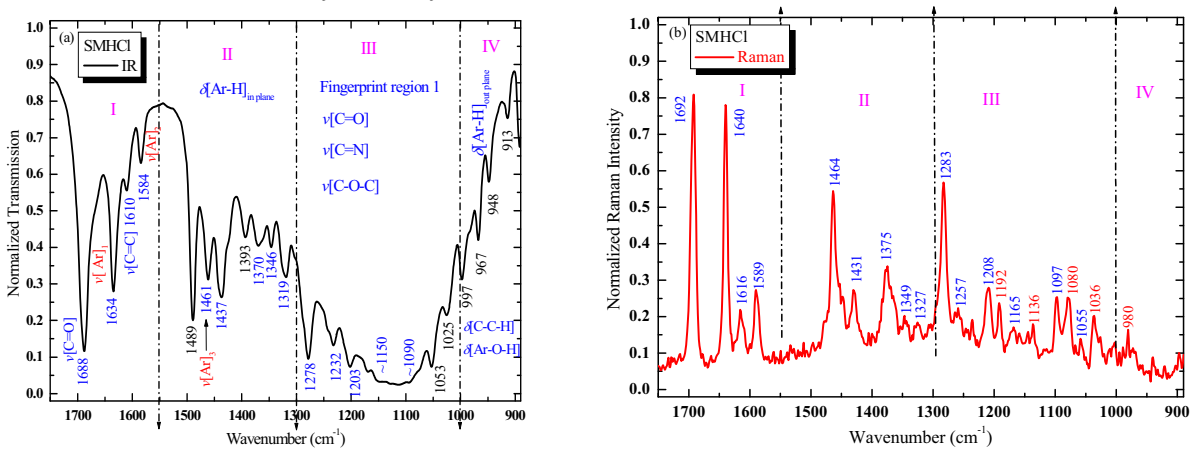

Fig. 2. IR (a) and Raman (b) spectra of the polycrystalline sinomenine hydrochloride $\left(\mathrm{SMHCl}, \mathrm{C}_{19} \mathrm{H}_{24} \mathrm{NO}_{4} \mathrm{Cl}\right)$ in the range of $1750-900 \mathrm{~cm}^{-1}$ (mostly the functional group region of [CC], [CO], [CN], etc). 
From Fig. 2, it can be shown that (1) all the five $v[\mathrm{CO}], v[\mathrm{CC}]$ and $v[\mathrm{Ar}]_{1 / 2 / 3}$ modes are both IR and Raman active; (2) only few $\delta[\mathrm{Ar}-\mathrm{O}-\mathrm{H}]$ and $\delta[\mathrm{Ar}-\mathrm{H}]_{\text {in/out-of plane }}$ modes are both $I R$ and Raman active because of unknown reasons.

In the range of 1300-600 $\mathrm{cm}^{-1}$ fingerprint region, as shown in Figs. 2 and 3, most of stretching modes $v[\mathrm{CO}], v[\mathrm{CN}]$ and $v[\mathrm{COC}]$ and bending modes $\delta[\mathrm{Ar}-\mathrm{O}-\mathrm{H}]$ and $\delta[\mathrm{Ar}-\mathrm{H}]_{\mathrm{in} / \text { out-of plane }}$ are both IR and Raman active, but distribute alternately so as to be distinguished with each other very difficultly. According to the comparison with the Raman spectral data of the SM [9], four bands of 308/334/354/374 $\mathrm{cm}^{-1}$ and two bands of $92 / 62 \mathrm{~cm}^{-1}$ appear jointly in the Raman spectra of both SM and $\mathrm{SMHCl}$, and can be tentatively, as we add a "?" in Fig. 3, assigned to be the stretching/bending $v / \delta[\mathrm{Ar}(\mathrm{OMe})]$ modes of the aromatic rings and $\mathrm{OCH}_{3}$ methoxyls, respectively. The $106 / 62 \mathrm{~cm}^{-1}$ bands appearing only in the SMHCl Raman spectrum are also assigned tentatively to be the stretching/ bending $v / \delta\left[(\mathrm{SMH})^{+} \mathrm{Cl}^{-}\right]$modes of the $(\mathrm{SMH})^{+}$ion as a whole and $\mathrm{Cl}^{-}$ion, respectively.
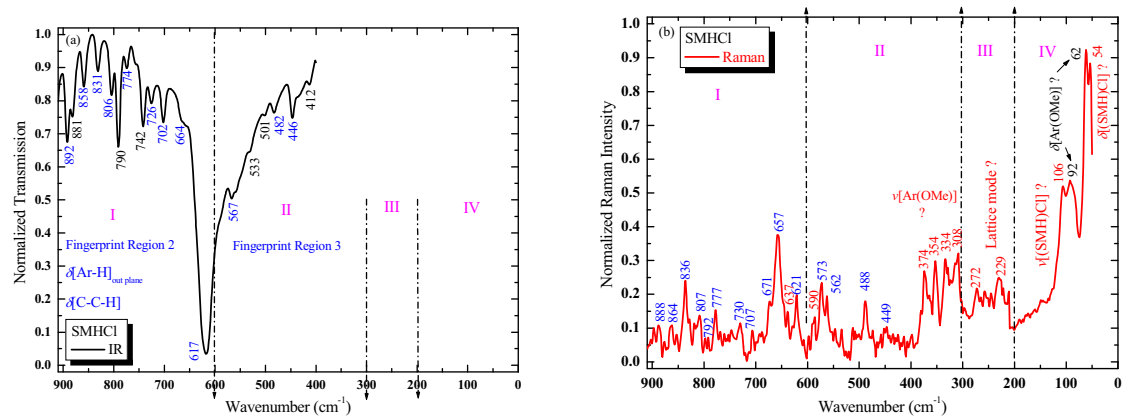

Fig. 3. (a) IR spectrum in the range of $900-400 \mathrm{~cm}^{-1}$ and (b) Raman spectrum in the range of $900-50 \mathrm{~cm}^{-1}$ (mostly fingerprint region) of the polycrystalline sinomenine hydrochloride $\left(\mathrm{SMHCl}, \mathrm{C}_{19} \mathrm{H}_{24} \mathrm{NO}_{4} \mathrm{Cl}\right)$.

\section{Summary}

We have measured high-resolution infrared and Raman spectra of the polycrystalline $\mathrm{SMHCl}$ to find out its whole really existing vibrational spectral bands. Except for the hydroxyl stretching modes $v[\mathrm{OH}]$ and IR active bands less than $400 \mathrm{~cm}^{-1}$, most normal vibration modes (about 34) are both IR and Raman active, additional 16 and 14 modes appear only in the IR and Raman spectra, respectively. In the mean while, 8 Raman bands less than $400 \mathrm{~cm}^{-1}$ are tentatively assigned to stretching/bending modes of the aromatic-ring-methoxyls and $(\mathrm{SMH})^{+}-\mathrm{Cl}^{-}$ions, respectively. More ideal one-to-one assignment of these IR and Raman bands needs intensive research work on many sinomenine derivatives.

\section{Acknowledgement}

This research was financially supported by the Scientific Research Foundation for the Returned Overseas Chinese Scholars, State Education Ministry of China ( $48^{\text {th }}$ batch, 2015). 


\section{References}

1. X.X. Zhao, C. Peng, H. Zhang, L.P. Qin, Sinomenium acutum: A review of chemistry, pharmacology, pharmacokinetics, and clinical use, Pharmaceutical Biol. 50 (2012) 1053- 1061.

2. F. Liao, Z.R. Yang, X.H. Lu, X.F. Guo, W.G. Dong, Sinomenine sensitizes gastric cancer cells to 5-fluorouracil in vitro and in vivo, Oncol. Lett. 6 (2013) 1604-1610.

3. T.S. Ma, G. Li, Z.H. Liu, H.J. Yang, G. Huang, L.C. Zhu, Study on purity of sinomenine hydrochloride crystal, China Pharmaceuticals, 14 (2005) 26-27 [in Chinese].

4. X.J. Lin, H. Zhang, S.G. Jin, W. Zhang, Preparation and in vitro Release of Sinomenine Hydrochloride-loaded Chitosan Nanoparticles, Chin. J. Exper. Trad. Med. Formulae, 17 (2011) 22-25 [in Chinese].

5. P. Dou, H. Zhang, W. Zhang, Optimization of Preparation Technology and Investigation of in vitro Release Mechanism of Sinomenine Hydrochloride-Polyvinyl Alcohol Microcapsules, Chin. J. Exper. Trad. Med. Formulae, 18 (2012) 49-52 [in Chinese].

6. W. Zhang, H.L. Fu, X.Y. Li, H. Zhang, N. Wang, W. Li, X.X. Zhang. Molecularly imprinted polymer doped with Hectorite for selective recognition of sinomenine hydrochloride, J. Biomater. Sci. - Polymer Ed. 27(2016): 144-156.

7. T.S. Ma, G. Li, Z.H. Liu, Z.X. Yang, G. Huang, Infrared spectrophotometry of sinomenine hydrochloride, Central South Pharmacy, 2 (2004) 108-110 [in Chinese].

8. X.D. Liu, J. Lu, M. Fujihala, D.D. Meng, et al. Local Laser Heating Effect on Raman Spectroscopy of $\beta-\mathrm{Co}_{2}(\mathrm{OH})_{3} \mathrm{Br}$, Adv. Mater. Res. 430-432 (2012) 566-569.

9. Information to be reported. 